\title{
Design of an Integrated Sensor Platform for Vital Sign Monitoring of Newborn Infants at Neonatal Intensive Care Units
}

\author{
Wei Chen $^{1 *}$, Sidarto Bambang Oetomo ${ }^{1,2}$, Loe Feijs ${ }^{1}$, Sibrecht Bouwstra ${ }^{1}$, \\ Idowu Ayoola ${ }^{1}$, Sietse Dols ${ }^{1}$ \\ ${ }^{1}$ Department of Industrial Design, Eindhoven University of Technology, $5612 \mathrm{AZ}$, \\ Eindhoven, The Netherlands \\ ${ }^{2}$ Neonatal Intensive Care Unit, Máxima Medical Center, 5500 MB, Veldhoven, \\ The Netherlands \\ Emails: \{w.chen, s.bouwstra, l.m.g.feijs\}@tue.nl, \{i.b.i.ayoola, \\ s.a.e.dols\}@student.tue.nl,s.bambangoetomo@mmc.nl
}

\begin{abstract}
Continuous health status monitoring and advances in medical treatments have resulted in a significant increase of survival rate in critically ill infants admitted into Neonatal Intensive Care Units (NICUs). The quality of life and long-term health prospects of the neonates depend increasingly on the reliability and comfort of the monitoring systems. In this paper, we present the design work of a smart jacket for vital sign monitoring of neonates at a NICU. The design represents a unique integration of sensor technology, user focus and design aspects. Textile sensors, a reflectance pulse oximeter and a wearable temperature sensor were proposed to be embedded into the smart jacket. Location of the sensor, materials and appearance were designed to optimize the functionality, patient comfort and the possibilities for aesthetic features. Prototypes were built for demonstrating the design concept and experimental results were obtained from tests on premature babies at the NICU of Máxima Medical Centre (MMC) in Veldhoven, the Netherlands.
\end{abstract}

Keywords: NICU, neonatal monitoring, sensor integration, smart textiles, reflectance pulse oximeter, design process

\section{INTRODUCTION}

Critically ill neonates are premature and full term infants that become severely ill during or immediately after birth. Premature infants are babies that are born after a pregnancy lasting 37 weeks or shorter. These neonates are extremely tiny and vulnerable to external disturbance. Continuous monitoring of health parameters is crucial for premature infants admitted at the neonatal intensive care unit (NICU) in hospitals, so that adequate and timely medical treatment can be instituted when acute health risks are detected. Advances in medical treatments resulted in a significant increase of survival [1,2].

${ }^{*}$ Corresponding author 
Vital signs for neonatal monitoring include body temperature, electrocardiogram (ECG), respiration and blood oxygen saturation [3, 4]. Presently, body temperature is monitored with adhesive thermistors; ECG and respiration are obtained by adhesive skin electrodes. The oxygen saturation of the blood is monitored by a transmissive pulse oximeter with the sensor applied to the foot or palm of the neonate [5]. Placement of these adhesive sensors and the presence of all the wires lead to discomfort and even painful stimuli when the sensors are removed. The disturbance, interruption of sleep, and lack of natural communication with parents all interfere with the babies' normal growth and development [6]. Since the long-term health prospects of the neonates depend more and more critically on reliable and comfortable health-status monitoring systems, alternative non-invasive monitoring of vital signs is important to provide convenient care, and hence may lead to improved developmental outcome of the neonates $[7,8]$.

Together with the development of the ambient intelligence concept [9], recent advances in sensor technologies and wireless communication technologies [10-13] enable the creation of a new generation of healthcare monitoring systems with wearable electronics and photonics. Smart textiles have been integrated into a garment for electrocardiogram (ECG) and respiration monitoring with wireless transmission [14, 15]. Reflectance pulse oximeters attached to the forehead [16, 17] have been developed. Non-invasive monitoring covers a wide range of applications, including healthcare, sports, work, entertainment, etc. For example, to improve the comfort of passengers by an adaptive entertainment system, non-invasive sensors for heart rate monitoring were embedded in aircraft passenger seats, enabling the emotion model manager to choose the proper music in a database for reducing the stress of passengers [18, 19]. Another example is to support economy class passengers to sleep well during a long haul flight. The sleeping posture of a passenger is detected by pressure sensors embedded in the aircraft seat, enabling the smart seat to provide support to the passenger [20, 21]. A user friendly EEG headset has been designed to enhance people's wellbeing based on biofeedback [22]. For the elderly, unobtrusive monitoring of sleeping patterns [23] and health status of people living alone at home [24] have been reported. Some early efforts and developments have been made towards non-invasive neonatal monitoring. For example, early design work of a future incubator and a biosensor belt for neonatal vital sign monitoring have been reported $[25,26]$. Dedicated design and integration are essential for achieving reliable neonatal monitoring with significantly improved patient comfort and ease of use.

Eindhoven University of Technology (TU/e) in the Netherlands has started a 10-year project on perinatology research in cooperation with the Máxima Medical Centre (MMC) in Veldhoven, the Netherlands. The goal of this research collaboration is to improve the healthcare of pregnant women and their children before, during and after delivery. The design skills needed range from medical science, human factors, material knowledge, smart textiles and form-giving to circuit design, user research, power management, signal processing and software engineering. Some intelligent designs have been developed covering different aspects of non-invasive perinatal monitoring, such as pregnancy monitoring [27-29], vital signs monitoring for neonates [30-32], data transmission [33], a device to support cardiopulmonary resuscitation of neonates [34, 
35], a power supply for neonatal monitoring [36, 37], neonatal behavioral state detection based on facial expression analysis [38] and delivery simulators [39, 40].

In this paper, we present the design work of a vital sign monitoring platform - Smart Jacket - integrated with non-invasive sensors for neonatal monitoring. The smart jacket aims at providing reliable health monitoring as well as a comfortable clinical environment for neonatal care and parent-child interaction. Textile sensors, a reflectance pulse oximeter, and a wearable temperature sensor are proposed to be embedded into the smart jacket for monitoring ECG, blood oxygen saturation and body temperature, respectively. Prototypes were built to demonstrate the performance of the design and obtain user feedback. The functionality, patient comfort and the possibilities for aesthetic features are taken into account for the sensor integration, materials and appearance design. Tests on premature babies at NICU of Máxima Medical Centre (MMC) show promising results of neonatal vital sign monitoring by the advanced integration of wearable sensors.

The rest of the paper is organized as follows: Section II explains the design process and design concept; Section III presents the integration of smart textile sensors for ECG measurements; Section IV demonstrates the integration of reflectance pulse oximeter for blood oxygen saturation monitoring; Section V presents neonatal body temperature measurements by wearable temperature sensor; and Section VI concludes the paper.

\section{DESIGN PROCESS AND DESIGN CONCEPT}

\subsection{Design Process}

Methodologies from the field of Industrial Design are applied in the design process, which involves a unique integration of knowledge from medical science, industrial design, sensor technology and electrical engineering. As shown in Figure 1, the iterative process begins with an information search that includes user research involving doctors and nurses of NICU at MMC, and gathering of information on users, clinical work domain analysis, technical feasibility and the design opportunities. Requirements are then derived from the information search, forming a base for brainstorm sessions which resulted in ideas about technological challenges, functionality issues within NICU as well as form-giving. Design choices are made through an iterative process in which proof of technology and user feedback provide valuable inputs for further development. Throughout the design process, the three aspects of Technology, User Focus and Design are strongly interwoven.

\subsection{Design Concept}

To achieve reliable, comfortable and user friendly neonatal monitoring, we envision a wearable platform integrated with unobtrusive sensors to replace the currently used adhesive skin sensors for neonatal vital sign monitoring. Based on the user research at NICU, the design should satisfy the following requirements:

- $\quad$ support the fundamental health monitoring functions and be safe to use in the NICU environments,

- be non-intrusive and avoid disturbance of the baby and avoid causes of stress,

- $\quad$ support continuous monitoring when the baby is inside the incubator or in their parents' hug during Kangaroo Mother Care (KMC) [41], 
- look friendly, playful and familiar to gain the feeling of trust for the parents and the caretakers,

- $\quad$ be easy to dress and undress.

The concept of the neonatal smart jacket is a non-invasive continuous monitoring platform embedded with wearable sensors, suitable for monitoring neonates inside the incubator and outside the incubator during KMC.

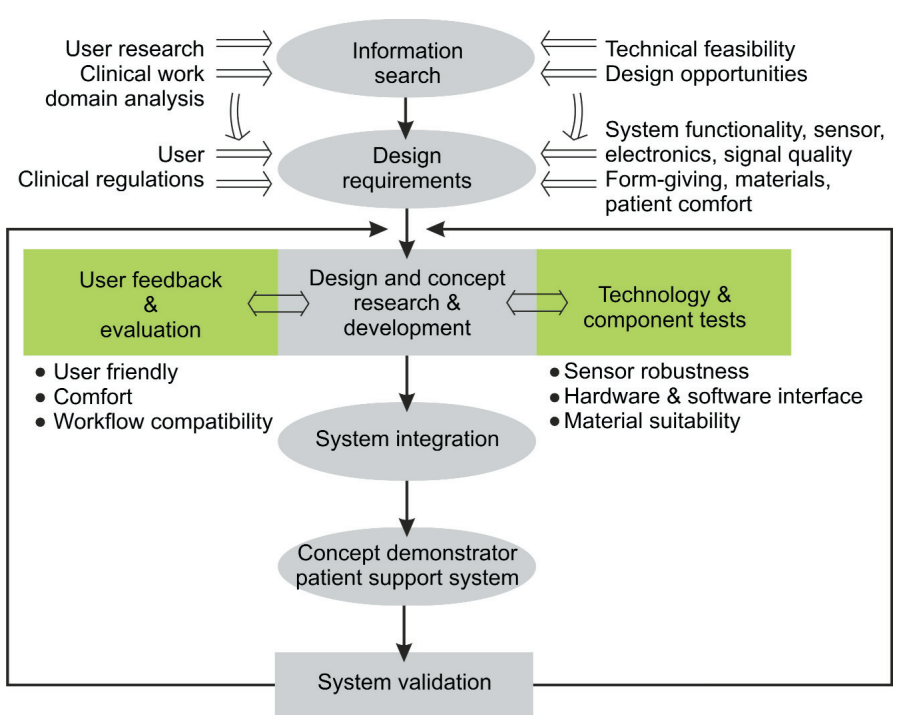

Figure 1. Design process.

Figure 2 shows a baby mannequin wearing the prototype smart jacket inside the incubator and during KMC. The jacket is open chest and has an open structure fabric on the back for phototherapy, clinical observation, and skin-to-skin contact during KMC. The jacket also consists of a hat with eye protection for phototherapy. When dressed on the neonate, the jacket just looks like a normal baby suit. The color combination of white and green with colorful happy animal heads is chosen to make it unisex while looking cheerful and clean.

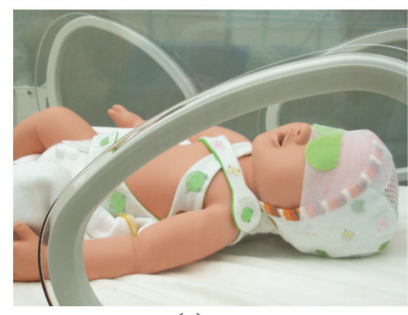

(a)

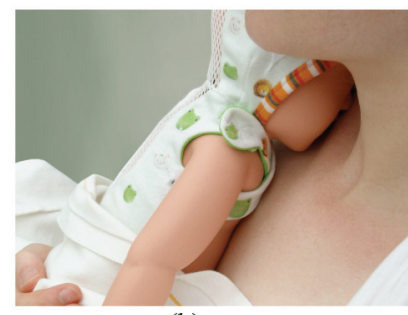

(b)

Figure 2. A baby mannequin wearing the smart jacket (a) inside the incubator, (b) during Kangaroo Mother Care. 
Different types of sensors are proposed to be embedded into the smart jacket for monitoring vital signs of neonates. In the following sections, we will present the design work on integrating textile sensors into the smart jacket for neonatal ECG monitoring, and the design work on a reflectance pulse oximeter and a compatible wearable temperature sensor to be integrated into the smart jacket. Prototypes and experimental results in clinical settings will be demonstrated.

\section{SMART TEXTILES FOR ECG MEASUREMENTS}

\subsection{Smart Textiles Integration}

Textile sensors are flexible, lightweight, non-irritating, and convenient to be integrated into the neonatal jacket for ECG and respiration monitoring. Two different textile electrodes were tested for the integration into the jacket: the silver coated Medtex $130+B$ textile electrodes by Shieldex ${ }^{\circledR}$ and the gold printed textile electrodes from TNO Science and Industry. Figure 3 displays the test patches with different versions of silver and gold textile electrodes and a blanket with large silver electrodes. The silver textile electrodes have a knitted structure of a nylon coated yarn with $99.9 \%$ silver metal. The nylon consists of $71 \%$ polyamide and $29 \%$ elastomeric fiber. Construction details can be seen in Fig. 4. Three layers of cotton are used, as shown in part (1) of Fig. 4. On the middle layer, the circuit is sewn with Shieldex® silver plated yarn, as shown in part (2). Part (3) shows that the electrode is sewn on the first layer, stitching through the circuit on the middle layer. The electrode's connection to the monitor is achieved by carbon wires from regular disposable gel electrodes. The ends of the carbon wires are stripped and sewn onto the circuit on the middle layer (see part 4). Finally the third cotton layer for isolation is sewn to the others (part 5). The gold printed textile electrodes consist of a thin smooth fiber with a metal print developed by TNO at Eindhoven, the Netherlands. The gold test patches are created in a similar way to the silver test patches, except that in future applications, the circuit and electrode can be printed in one piece.

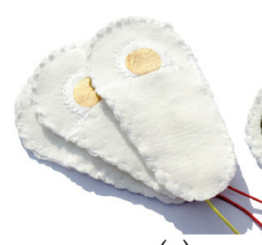

(a)

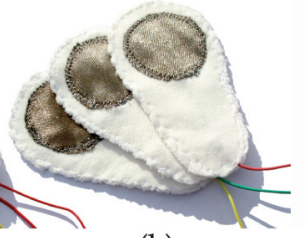

(b)

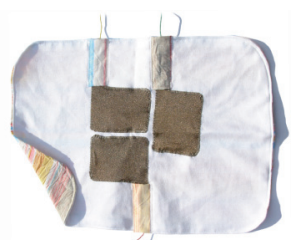

(c)

Figure 3. Test patches with different versions of silver and gold textile electrodes ( $a$ and b), and a blanket with large silver electrodes (c). 


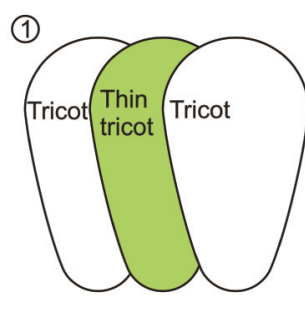

(4)

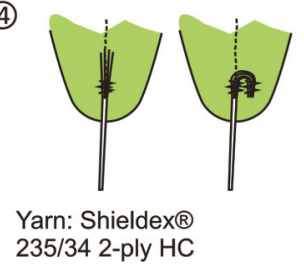

(2)
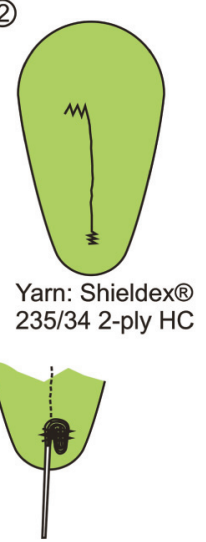

(3)

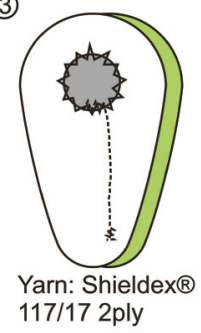

(5)

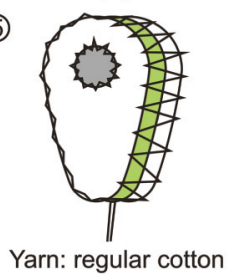

Figure 4. Construction of textile electrodes.

Figure 5 shows the silver coated textile electrodes integrated into the prototype smart jacket in the same manner as in Fig. 4. To assure comfort, care is taken to prevent thick seams and sharp edges, and cotton is chosen for its softness and breathability. The wiring was implemented with conductive yarn, which is silver coated nylon from Shieldex®. The six textile electrodes are located at different positions in the jacket to perform diversity measurements. If one sensor becomes loose from the skin, another sensor can provide a better signal. By this diversity measurement method, unstable signals caused by movement artifacts can possibly be reduced by always choosing the optimal signal for further processing. The textile electrodes are connected only on one side, in order to allow stretch of the jacket without stretch of the sensor itself.

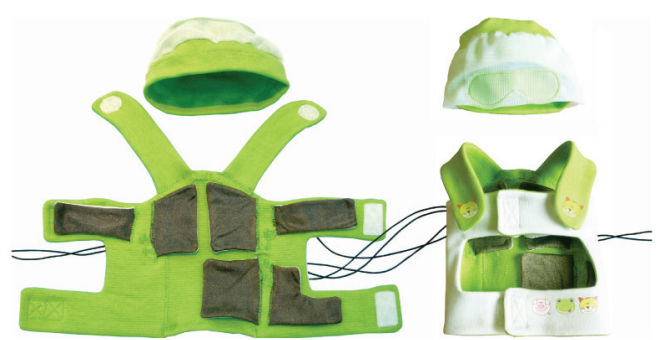

Figure 5. Prototype smart jacket. 


\subsection{Clinical Testing}

Several experiments of explorative nature were carried out with neonates in the NICU at MMC. The first test is a comparison of different types of electrode test patches, and the second on wearability of the jacket. An analysis of risks was performed before applying the smart jacket prototypes to the NICU. Together with clinical physicists, a hospital hygiene and infection expert, and a neonatologist, the safety of the monitoring system and hygiene and allergy risks were analyzed. The most critical dimensions are: (1) leakage current which has to meet the CF class 1 standard, (2) hygiene and infection risks, for which the hospital protocol standards have to be met which consists of thermo disinfection washing at 60 degrees and cleaning plastics with alcohol, and (3) allergenic reactions which can be tested by evaluation of a subject's skin after wearing test samples for 48 hours. The prototype stood the CF class 1 and the allergy tests, and has been cleaned according to hospital hygiene standards. For the 24 monitoring scenario, the jacket embedded with textile electrodes will be worn for a maximum of 48 hours according to the hospital hygiene standards, and after that the jacket will be washed. The ethical commission of the MMC approved the experiments.

Figure 6 shows the test setup. With the consent of the parents, the textile electrode patches were tested with two subjects: one neonate of 30 weeks and 5 days and the other of 31 weeks and 6 days, both admitted in the NICU at MMC. The ECG was sensed by three textile electrodes in regular configuration and the data was acquired with a GE Healthcare Solar® $8000 \mathrm{M}$. The unprocessed digital data of derivation II was obtained from a network and imported and filtered in MATLAB. Filtering technologies for ECG analysis has been reported in $[42,43]$. We applied a notch, a 2-Hz Butterworth high pass and a $70-\mathrm{Hz}$ Butterworth low pass filter to remove the $50-\mathrm{Hz}$ and higher harmonics, base line wandering and high frequency noise, respectively.

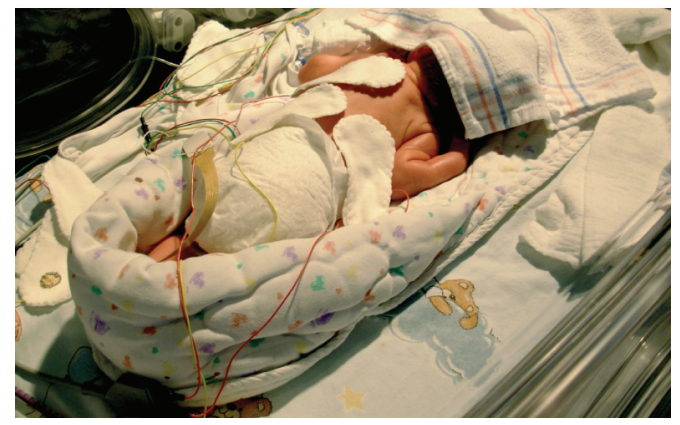

Figure 6. Test setup.

We evaluated the quality of the ECG signals obtained by textile electrodes varying in material, size, and applied pressure, and qualitatively compared it to the golden standard of gel electrodes (3M $\left.\mathrm{M}^{\mathrm{TM}} 2282 \mathrm{E}\right)$. The signals were recorded in series. In two observations, we assessed whether (a) the signals were stable, (b) the QRS complex features could be distinguished, and (c) the signals could cope with movement artifacts. 
The QRS complex refers to the deflections in the tracing of an ECG, comprising the Q, $\mathrm{R}$, and $\mathrm{S}$ waves, that corresponds to the depolarization of the ventricles.

Table 1 summarizes the comparison of different electrodes. It shows that the large silver electrodes of $80 \mathrm{~mm} \times 60 \mathrm{~mm}$ offer a stable ECG signal with low noise under the condition that pressure is applied by the baby's weight (e.g., baby lies on the blanket with textile electrodes as shown in Figure 3). Small silver electrodes (diameter D = 16 $\mathrm{mm}$ ) seem to require a higher pressure in order to make proper contact. The silver electrodes are hypoallergenic and do not change properties considerably after a few washing cycles. The small gold printed electrodes obtain a stable ECG signal with low noise. A little pressure applied by hand is required for the electrode to establish initial contact. After letting go, the contact maintains. The gold print textile electrodes are not hypoallergenic and lose conductivity after washing, due to corrosion of the metal layer beneath the gold. Therefore, the most promising textile electrodes for the smart jacket are (1) large ( $\mathrm{D}=40 \mathrm{~mm})$ silver plated textile electrodes, and (2) small ( $\mathrm{D}=16 \mathrm{~mm})$ gold printed electrodes.

Table 1. Qualitative comparison of different electrodes.

\begin{tabular}{|c|c|c|c|c|}
\hline $\begin{array}{c}\text { Type of } \\
\text { electrode }\end{array}$ & $\begin{array}{c}\text { Stability } \\
\text { (breathing } \\
\text { movements) }\end{array}$ & $\begin{array}{c}\text { QRS } \\
\text { complex } \\
\text { features }\end{array}$ & $\begin{array}{c}\text { Skin } \\
\text { contact }\end{array}$ & $\begin{array}{c}\text { Stability } \\
\text { (limb } \\
\text { movements) }\end{array}$ \\
\hline $3 \mathrm{M}^{\mathrm{TM}} 2282 \mathrm{E}$ & $\bullet \bullet \bullet \bullet$ & $\bullet \bullet \bullet \bullet$ & $\bullet \bullet \bullet \bullet \bullet$ & $\bullet \bullet \bullet \bullet \bullet$ \\
\hline $\begin{array}{c}\text { Gold print, D } \\
\text { =16mm }\end{array}$ & $\bullet \bullet \bullet$ & $\bullet \bullet \bullet \bullet \bullet$ & $\bullet \bullet \bullet \bullet$ & $\bullet \bullet \bullet \bullet$ \\
\hline $\begin{array}{c}\text { Silver } \\
\text { Medtex } \\
80 \mathrm{~mm} \times\end{array}$ \\
$\begin{array}{c}60 \mathrm{~mm}, \text { with } \\
\text { baby weight }\end{array}$
\end{tabular}

Figure 7 plots the recorded measurements of gold printed textile electrodes and silver Medtex $80 \mathrm{~mm} \times 60 \mathrm{~mm}$ electrodes when the baby lies still. It is shown that the QRS complex is clearly visible from the ECG obtained by both type of textile electrodes.

With this platform, we aim at the monitoring of vital functions similar to the conventional monitoring system using adhesive electrodes. The monitor is used as an indicator of potential harmful events by acoustic alarming, and for the screening of heart rhythm disturbances and ECG complex abnormalities. It is not our intention to replace with the present system the more accurate conventional standard ECG for diagnostic purposes. 

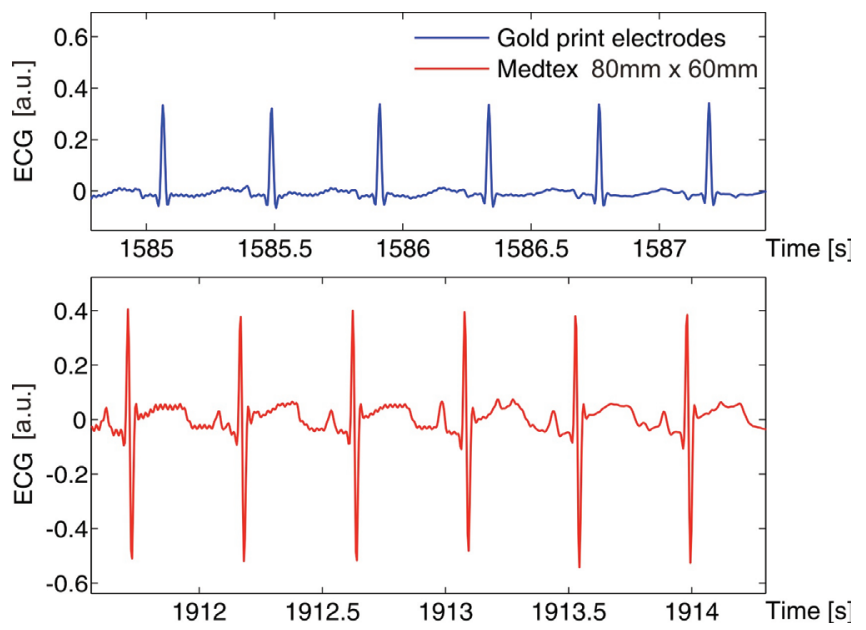

Figure 7. Signals obtained from gold printed and Medtex $80 \mathrm{~mm} \times 60 \mathrm{~mm}$ electrodes.

Due to the nature of conductive textiles, the quality of the ECG signal obtained with textile electrodes cannot exceed that of the gel electrodes as textile electrodes are 'dry' electrodes with higher skin resistance and have a flexible structure that causes artifacts. We are currently performing experiments in clinical settings to understand the source of movement artifacts. In the development of the diverse measurement method, we are aiming to improve the reliability of the textile electrodes under relative little movement and constant pressure by weight offered within the specific context of a NICU.

Apart from reliable technology, the success of the smart jacket largely depends on the wearable comfort of the jacket. Tightness is desirable for sensor contact, although it might be in conflict with wearable comfort. Therefore, extra caution is taken by performing a wearability test in an early design stage. Figure 8 shows a stable neonate of 34 weeks being dressed in the first prototype of the smart jacket while being filmed. The dressing process of the smart jacket went very smoothly. The dressing time was about one minute. The model needs to be more adjustable in size due to large variations in proportions and range of dimensions, as in the NICU, neonates can grow from $500 \mathrm{~g}$ to $2000 \mathrm{~g}$ and body proportions vary, especially when caused by medical conditions.

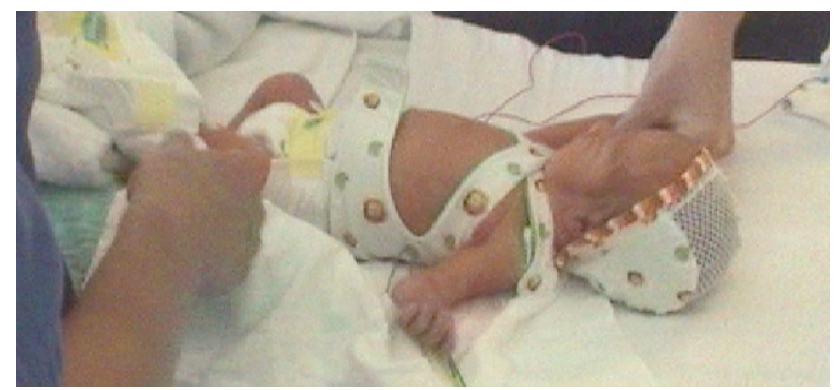

Figure 8. Wearability test with the first smart jacket prototype. 
Based on interviews with parents and medical staff, a conclusion can be drawn that the user groups are positive about the early design. They especially appreciate the freedom of movement, the aesthetic design, the integrated eye-protection, and the stress-less dressing process.

\section{REFLECTANCE PULSE OXIMETER FOR BLOOD OXYGEN SATURATION MONITORING}

\subsection{Integration of Reflectance Pulse Oximeter}

Blood oxygen saturation is one of the key parameters for health monitoring of premature infants at an NICU. There are two non-invasive techniques based on Near Infrared Spectroscopy (NIRS) for pulse oximetry $\left(\mathrm{SpO}_{2}\right)$ measurement: transmissive and reflective techniques $[16,44]$. Currently, the transmissive technique is used in hospitals, which works on thin body parts, such as the earlobe, finger tip, neonate's palm and foot. For the transmissive technique, the light emitters and photodiode have to be placed on opposite sides of a peripheral pulsating vascular bed. Alternatively, the reflective technique is able to sense the red and infrared light absorption on a single surface with the light emitters and photodiode placed side by side. The reflectance pulse oximetry provides the flexibility for measurements at different locations on the neonates, and has the potential to be integrated into a non-invasive monitoring platform, such as the smart jacket. The design, integration and prototype of the reflectance $\mathrm{SpO}_{2}$ system are described below.

The block diagram of the $\mathrm{SpO}_{2}$ system design is shown in Figure 9. It consists of a sensor head, a wireless module and a monitor. The sensor head is a reflectance pulse oximeter which includes a red-infrared emitter and a photo diode integrated into a textile. The wireless module is used as a local processor which sends signals wirelessly to the monitor. It is also used to modulate and power the sensor components. A standard oximetry device (Avant ${ }^{\circledR} 4000$ Wireless Tabletop Pulse Oximetry by Nonin) was employed for wireless transmission and advanced $\mathrm{SpO}_{2}$ processing.

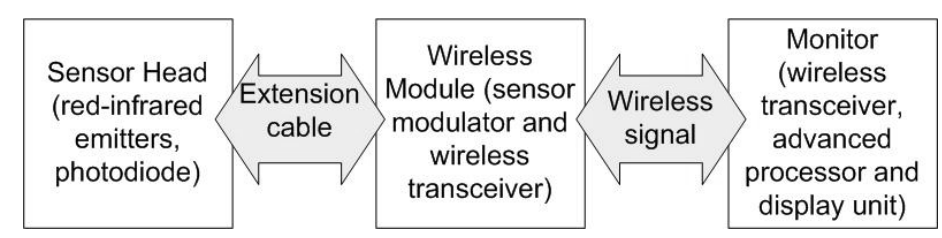

Figure 9. Block diagram of the $\mathrm{SpO} 2$ system design.

The connection circuit of the sensor head which consists of a photo diode, red and infrared light emitters, and integrated switch, is depicted in Fig. 10. The polarities of the red-infrared light emitter are connected to the wireless module independently from the photo diode, and a switch circuitry is embedded to activate the device when plugged in. 


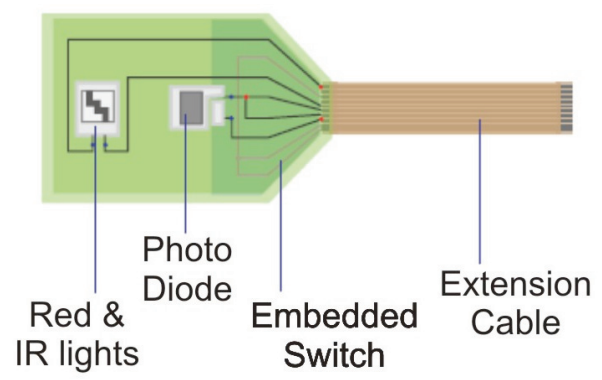

Figure 10. Connection circuit of sensor head, which includes a photo diode, Red \& Infrared emitters and integrated switch.

Part of the functional aspects of the design is to satisfy safety, comfort, and reliability criteria. A topography for the layout of sensors, connectors, and wires was made to satisfy these criteria. Different layers were used for insulation, protection, and other functions. Figure 11 illustrates the layer hierarchy, and assembled layers and components. Depicted in Figure 11 (a), layer 1 is the top layer which has direct contact with the skin. It is made of cotton with a few silicon spots which act as an anti-slip to minimize false alarms caused by movement artifacts. Layer 2 is a transparent layer made of Nylon or transparent plastic which is not reactive and does not deflect light. Layer 2 is used to protect the surface of the electrodes and prevents direct contact of the electrodes with the patient's skin. Layer 3 is a porous and semi-elastic material which holds the electrodes and the connection circuitry unto the textile. Layer 4 holds the extension cable which connects to the wireless module, while layer 5 serves as an insulator between layers 4 and 6. A switch is integrated on layer 6 for automatic switching when plugged and layer 7 insulates the layer from exposed conductors. Various connection and manufacturing techniques were explored to maximize the sensitivity of the electrodes.

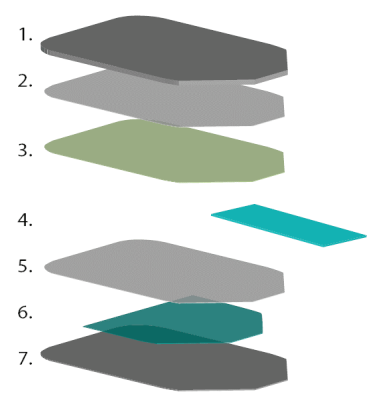

(a)

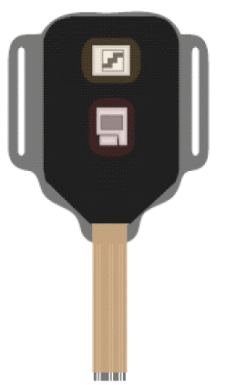

(b)

Figure 11. (a) Layer hierarchy for integrating the device sensor into the neonatal jacket: 1. Top layer, 2. Protection layer, 3. Component Layer, 4. Extension layer, 5. Isolation layer, 6. Switch layer, 7. Base layer. (b) Assembled layers and components. 
Prototypes were manufactured to demonstrate the functionality and design concept and to test the performance of the monitoring device. The extension cable plus sensor head and the internal structure of the sensor head are displayed in Fig. 12. The LED acts as the light emitter while the photo diode was sensitive to only red and infrared lights, which detects the intensity of the reflected lights. Materials used were flexible to enhance the comfort of the design and to enhance compatibility for integration into the smart jacket. To increase robustness of the design, effective conductive, insulating and connective materials were used. For example, a $0.5 \mathrm{~mm}$ insulated copper wire and soldering iron were used to ensure reliable connections; and a $0.1 \mathrm{~mm}$ flex wire was used for the extension cable. All layers were integrated into this prototype, but layer 3 and 6 in Fig. 11 were merged together.

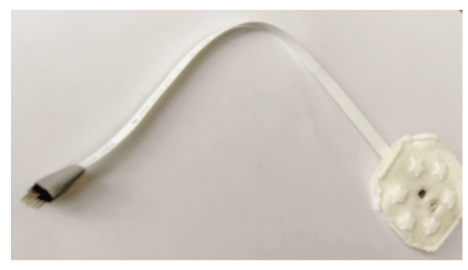

(a)

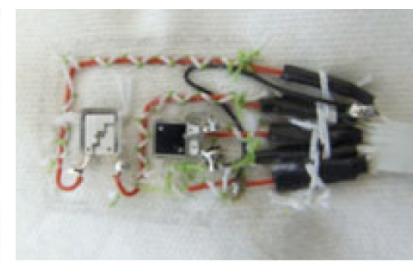

(b)

Figure 12. (a) Picture of the extension cable and the sensor head of the second prototype with the top layer made of soft cotton with silicon spots. (b) The internal structure of the sensor head.

\subsection{Clinical Testing}

The goal for conducting a user-test on babies in the incubator of the NICU is to validate the performance of the pulse oximeter prototype and explore opportunities for placing the sensors on the body for a proper integration into the jacket. The focus was to determine positions in which the sensor could read the $\mathrm{SpO}_{2}$ and heart rate signs accurately. The tests were conducted at the NICU of MMC Veldhoven, the Netherlands.

To validate the test results, a standard patient monitor Solar® $8000 \mathrm{M}$ was employed and the $\mathrm{SpO}_{2}$ and heart rate readings from the prototype and the standard patient monitor were compared to verify the results of the test. Participants for the user-test involved a medical doctor, an NICU nurse, a technical expert, a designer/researcher, and test personnel. Before implementing the test on the baby, the prototype was first tested on an adult to ensure that it was working properly and then sterilized for clinical testing.

The device was tested on a premature infant, born after 30 weeks gestation in stable health condition. A neonatologist and an NICU nurse from MMC were present during the testing. With the permission of the hospital and the parents, the prototype was placed and observed on various locations of the neonate's body. Examples of the tested positions were on the back, belly, chest, sides, legs, arm, neck and various locations on the head. Figure 13 shows an instance of the test on a baby inside an incubator in the NICU. 


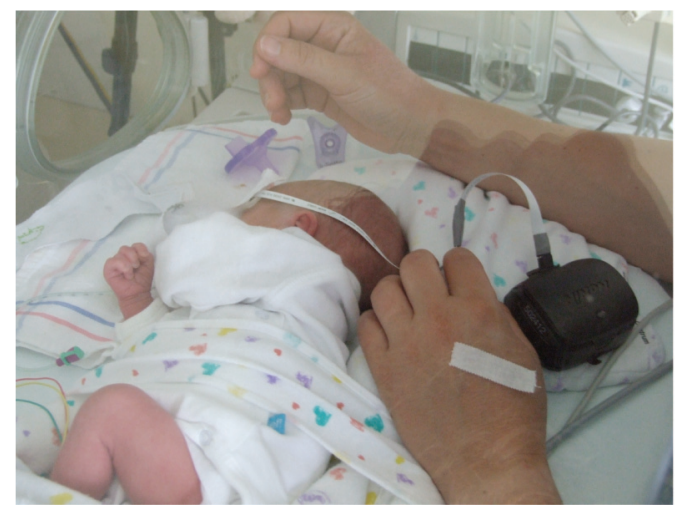

Figure 13. Clinical test on a baby inside an incubator in the NICU at MMC.

Through the pilot study, we found that the results obtained at the forehead, back of the head, back of the shoulders, neck, and foot best agreed with readings of the standard monitor. Sample prototype test results on the forehead are compared with the readings of the standard patient monitor in Fig. 14, demonstrating very good agreement. The consistent measurements on different positions were verified on another premature NICU patient. Therefore, these positions (forehead, back of head, back of shoulders, neck, and foot) are recommended for further research on the sensor locations, followed by detailed performance evaluation including quantitative error analysis, signal-tonoise ratio comparison, etc.

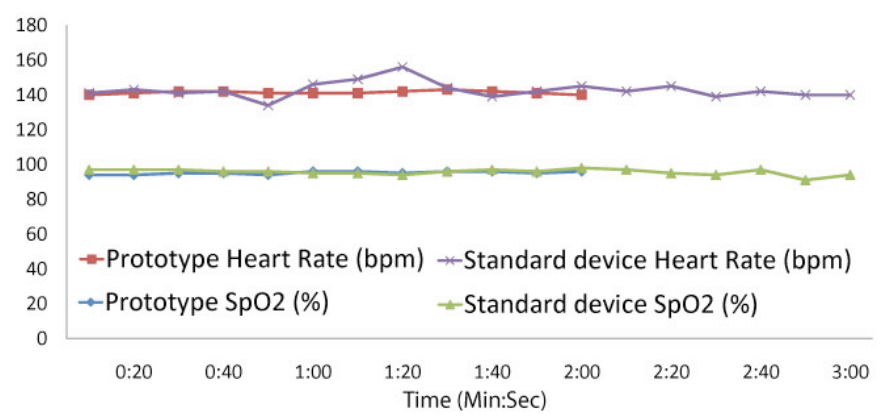

Figure 14. Comparison between prototype and standard device readings on a baby's forehead in the NICU.

\section{WEARABLE SENSORS FOR NEONATAL BODY TEMPERATURE MONITORING}

\subsection{Integration of Body Temperature Sensor}

Body temperature serves as an indicator for impeding infections and general stability of the infant, and is therefore, one of the vital signs for continuous neonatal monitoring. 
Temperature is typically measured at one site on the body. Moreover, the parameter is used to determine adequate environmental temperature for an optimal growth and development of neonates. Currently in hospitals, transcutaneous thermometry [45] is employed for core temperature monitoring of neonates. A thermistor is placed between the infants back and the mattress of the incubator, attached to the skin with a foam adhesive disk insulator.

In this section, we present a design for monitoring the body temperature of NICU neonates with a negative temperature coefficient (NTC) sensor integrated into a soft belt, which has potential to be incorporated into a non-invasive neonatal monitoring platform, such as the smart jacket. The soft belt is made of bamboo fabric, with a small integrated thermistor (see Fig. 15). To optimize the functionality and patient comfort, relevant practical issues are studied and considered in the design, such as location of the sensor, conductive textile wires for the connection, choice of materials, and appearance of the prototype.

The prototype belt, includes an NTC Mon-A-Therm 90045 temperature sensor $(2 \mathrm{~mm} \times 3 \mathrm{~mm})$. According to the NICU doctors, the sensor was located above the liver to provide optimal body temperature measurements. The temperature sensor is isolated using soft cotton foam, as shown in Fig. 16, to limit external influences such as the room temperature. Cotton foam was chosen because it is flexible, soft, and comfortable to wear. Similar method of isolation is employed for the Mon-A-Therm 90045 medical temperature sensor at hospital.

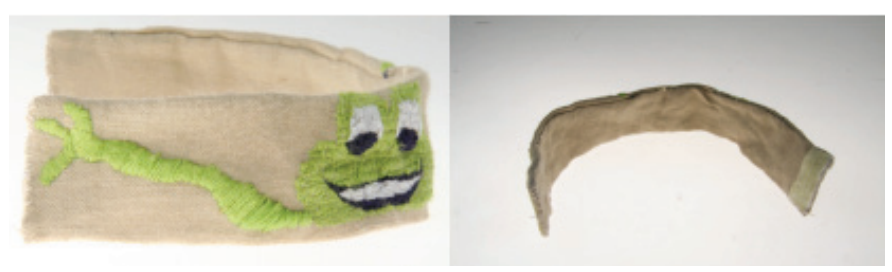

Figure 15. The prototype belt for monitoring body temperature.

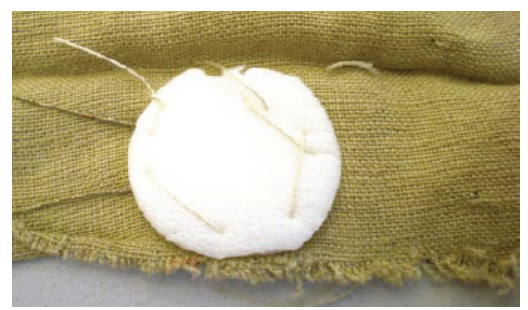

Figure 16. The temperature sensor isolated by cotton foam. 
The design moves sensors and foam to an invisible background for a user friendly interface and better parent-children bonding, and the happy frog appearance is chosen for aesthetic purposes. There are no adhesive components and hard wires in the belt, and instead, flexible and soft conductive textile wires made of Shieldex® silver plated nylon yarn are applied for connections. Bamboo textile was chosen as the material for the prototype belt because they are soft, breathable and therefore comfortable to wear.

\subsection{Clinical Testing}

Performance validation and evaluation of the proposed design are important parts of the design process. Various experiments were carried out to test the functionality and behavior of the prototype belt, and some other tests focused on sensors and conductive textiles for the prototype belt. The results showed that the electrical resistance of the conductive nylon yarn is higher than the regular copper wire. After calibration, stable measurements can be achieved. The experiments and user tests consist of several phases. First of all, the NTC temperature sensor in combination with the conductive textile wires was calibrated. Second, the measurement accuracy of the prototype belt was validated by tests on a premature baby in the incubator of the NICU.

The prototype belt was tested at the NICU of MMC Veldhoven, on a premature infant born after 30 weeks of gestation in stable health condition. A neonatologist and an NICU nurse from MMC were present during the testing. After obtaining permission of the parents, the prototype belt was placed on the neonate's body. Figure 17 shows the baby inside an incubator during the test in the NICU at MMC Veldhoven.

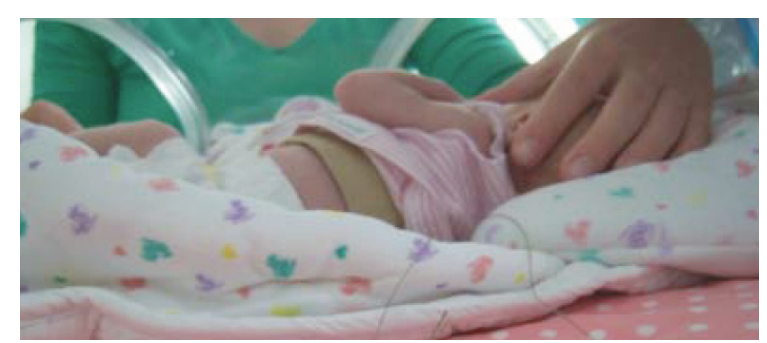

Figure 17. Testing of the prototype belt on a neonate in the NICU at MMC, The Netherlands.

Body temperature was measured in degrees Celsius by both a conventional NICU sensor connected to the Solar® $8000 \mathrm{M}$ patient monitor and the prototype belt with NTC sensor integrated and conductive textile wires as connector. Data were collected for 20 minutes after the sensor reading stabilized. The time interval between two consecutive readings was about 2 minutes. We found that the error of the prototype belt reading was within $0.1^{\circ} \mathrm{C}$. Further research will be conducted to quantitatively analyze the performance of the prototype belt. 


\section{DISCUSSION}

The challenges in developing the new generation of neonatal monitoring stem from both integration of technologies for system performance enhancement and user-friendly design for comfort improvement. Moreover, the NICU environment involves the participation of multiuser (e.g., caretakers, parents, and critically ill neonates). The users' expectations and requirements must be collected and balanced by a structured design process. The introduction of new technologies in such a critical setting has to address a set of different issues, such as acceptance of the design from the medical personnel and families, understanding how a new system or a new device can be integrated into the pre-existing work practice, how it can support new "emerging" care procedures, and how this is perceived by the users. Therefore, multidisciplinary cooperation from the fields of medical science, clinics, industrial design, electrical engineering and software engineering are needed throughout the design process. The design opportunities inspire TU/e researchers from different departments and medical staff from MMC to work together towards the future of neonatal monitoring solutions.

Before the smart jacket can be employed for the routine monitoring of neonates at NICU, extensive experiments and clinical trials need to be conducted to ensure the robustness of the system, the quality of the monitored signal, the usability, and the comfort of the design. For example, the dynamic recombination of the ECG sensors is a promising approach to counteract the movement artifacts and achieve more stable signals for improving the system reliability. Experiments are carried out in clinical settings to understand the source of movement artifacts in order to transfer the knowledge into the design and to create an automatic diversity measurement method.

For the future work, the research on the smart jacket sensory platform will be continued, first by further development of the ECG sensors, $\mathrm{SpO}_{2}$ sensors, wireless transmission and an adjustable size for different patients to enable clinical reliability tests. All vital sign monitoring functions will be integrated into the smart jacket by design for improving the neonates' comfort. Measurements and experiments need to be conducted at the NICU of MMC and other hospitals to assess the performance of the design. Protocols of evaluating the new monitoring devices on neonates need to be developed together with other experts in neonatal monitoring. Users' participation and feedback will be always taken into account during the iterative design process. Furthermore, statistical evidences about the effectiveness of the monitoring provided by the prototype need to be analyzed for the design evaluation.

\section{CONCLUSION}

The future of neonatal monitoring requires reliability of the system, non-invasiveness of sensors, comfort, and ease of use. The work presented in this paper illustrated the ongoing research on the design of a neonatal smart jacket as an integrated sensor platform for vital sign monitoring at an NICU. An iterative design process has been applied to conduct user research, concept generation, technology integration, prototype implementation, experimental testing and user feedback. Prototypes were built and tested to demonstrate the monitoring of ECG, blood oxygen saturation and body temperature with wearable sensors. The concept, prototype and promising testing results showed the progress in the integration of non-invasive sensors into the future incubator. 


\section{ACKNOWLEDGMENT}

The authors would like to thank Geert van den Boomen, Willem Jin, Hanneke Hooft van Huysduynen, and Karlijne Schoot, from the department of Industrial Design, Eindhoven University of Technology, the Netherlands, for their precious contributions and collaboration on the neonatal monitoring project. We would like to thank TNO Science and Industry, the Netherlands, for providing the technology and materials to substantiate the gold printed electrodes. We would like to thank Dr. Jeroen Veen and Dr. Martijn Schellekens from Philips Research Eindhoven for their instrumental support to the $\mathrm{SpO}_{2}$ sensors, both in material and technical solutions. We also thank Statex ${ }^{\circledR}$ for supplying the Shieldex ${ }^{\circledR}$ silver coated textiles and yarns. Furthermore, our special thanks go to the 'Textile Management' department at the Saxion Hogeschool Enschede and to the baby fashion magazine 'Knippie Baby', for sharing their knowledge on textiles and fashion. We are particularly grateful to Ms. Astrid Osagiator together with the medical staff at MMC Veldhoven and the parents of babies in the NICU for all their support and valuable inputs.

\section{REFERENCES}

[1] K. Costeloe, E. Hennessy, A. Gibson, N. Marlow, and A. Wilkinson, The EPICure study: outcomes to discharge from hospital for infants born at the threshold of viability, Pediatrics, 2000, 106, 659-671.

[2] M. De Kleine, A. den Ouden, L. Kollee, A. Ilsen, A. van Wassenaer, R. Brand, and S. VerlooveVanhorick, Lower mortality but higher neonatal morbidity over a decade in very preterm infants, Paediatric and Perinatal Epidemiology, 2007, 21, 15-25.

[3] H. Als, G. Lawhon, E. Brown, R. Gibes, F. Duffy, G. McAnulty, and J. Blickman, Individualized behavioral and environmental care for the very low birth weight preterm infant at high risk for bronchopulmonary dysplasia: neonatal intensive care unit and developmental outcome, Pediatrics, 1986, 78, 1123-1132.

[4] R. A. Polin and W. W. Fox, Fetal and Neonatal Physiology, W. B. Saunders Company, 1992.

[5] I. Murković, M. Steinberg, and B. Murković, Sensors in neonatal monitoring: Current practice and future trends, Technology and Health Care, 2003, 11, 399-412.

[6] H. Als, L. Gilkerson, F. Duffy, G. Mcanulty, D. Buehler, K. Vandenberg, N. Sweet, E. Sell, R. Parad, and S. Ringer, A three-center, randomized, controlled trial of individualized developmental care for very low birth weight preterm infants: medical, neurodevelopmental, parenting, and caregiving effects, Journal of Developmental \& Behavioral Pediatrics, 2003, 24, 399-408.

[7] W. Chen, S. Bambang Oetomo, and L. Feijs, Neonatal Monitoring: Current Practice and Future Trends, in Handbook of Research on Developments in e-Health and Telemedicine: Technological and Social Perspectives: IGI Global, 2010.

[8] W. Chen, S. Bouwstra, S. Bambang Oetomo, and L. Feijs, Intelligent Design for Neonatal Monitoring with Wearable Sensors, in Intelligent and Biosensors: INTECH, 2010, 386 - 410.

[9] E. H. L. Aarts and J. L. Encarnação, True visions: The emergence of ambient intelligence (2nd ed.): Springer, 2008.

[10] A. Goldsmith, Wireless communications: Cambridge Univ Pr, 2005.

[11] X. Tao, Wearable electronics and photonics: Woodhead, 2005.

[12] G. Yang and M. Yacoub, Body sensor networks: Springer-Verlag New York Inc, 2006.

[13] L. van Langenhove, Smart textiles for medicine and healthcare: materials, systems and applications: Woodhead Publishing, 2007.

[14] M. Catrysse, R. Puers, C. Hertleer, L. Van Langenhove, H. Van Egmond, and D. Matthys, Towards the integration of textile sensors in a wireless monitoring suit, Sensors and Actuators A: Physical, 2004, $114,302-311$. 
[15] R. Paradiso, G. Loriga, and N. Taccini, A wearable health care system based on knitted integrated sensors, IEEE Transactions on Information Technology in Biomedicine, 2005, 9, 337-344.

[16] Y. Mendelson, R. Duckworth, and G. Comtois, A wearable reflectance pulse oximeter for remote physiological monitoring, in 28th Annual International Conference of the IEEE Engineering in Medicine and Biology Society (EMBS), 2006, 912-915.

[17] New OxyAlert ${ }^{\mathrm{TM}}$ NIRSensors, from http://www.somanetics.com/Invos-Infant-Neonatal.asp.

[18] H. Liu, J. Hu, and G. W. M. Rauterberg, LsM: A New Location and Emotion Aware Web-based Interactive Music System, in Proceedings of IEEE International Conference on Consumer Electronics (ICCE 10) Piscataway NJ.: IEEE.

[19] H. Liu, J. Hu, and G. W. M. Rauterberg, Music Playlist Recommendation Based on User Heartbeat and Music Preference, in Proceedings of 2nd IEEE 2009 International Conference on Computer Technology and Development(ICCTD '09): IEEE press, 2009.

[20] C. Tan, W. Chen, F. Kimman, and G. Rauterberg, Sleeping Posture Analysis of Economy Class Aircraft Seat, in World Congress on Engineering, London, U.K., 2009, 532-535.

[21] C. Tan, W. Chen, H. Liu, and M. Rauterberg, Adaptive Framework and User Preference Modeling for Economy Class Aircraft Passenger Seat, in Third UKSim European Symposium on Computer Modeling and Simulation, Athens, Greece, 2009, 66-69.

[22] J. van Aart, E. Klaver, C. Bartneck, L. Feijs, and P. Peters, EEG headset for neurofeedback therapy : enabling easy use in the home environment, in International Conference on Bio-inspired Signals and Systems, 2008, 23-30.

[23] A. Adami, T. Hayes, and M. Pavel, Unobtrusive monitoring of sleep patterns, in 25th Annиal International Conference of the IEEE EMBS, 2003, 1360-1363.

[24] A. Kaushik and B. Celler, Characterization of PIR detector for monitoring occupancy patterns and functional health status of elderly people living alone at home, Technology and Health Care, 2007, 15, 273-288.

[25] A. Rullo, P. Marti, E. Grövall, and A. Pollini, End-user composition and re-use of technologies in the Neonatal Intensive Care Unit, in Proceedings Pervasive Healthcare, 2006.

[26] L. Piccini, O. Ciani, E. Grövall, P. Marti, and G. Andreoni, New monitoring approach for Neonatal Intensive Care Unit, in 5th International Workshop on Wearable Micro and Nanosystems for Personalised Health, 2008.

[27] R. Vullings, C. Peters, R. Sluijter, M. Mischi, S. Oei, and J. Bergmans, Dynamic segmentation and linear prediction for maternal ECG removal in antenatal abdominal recordings, Physiological Measurement, 2009, 30, 291-307.

[28] C. Rabotti, M. Mischi, J. Laar, G. Oei, and J. Bergmans, Estimation of internal uterine pressure by joint amplitude and frequency analysis of electrohysterographic signals, Physiological Measurement, 2008, 29, 829-841.

[29] S. Martens, C. Rabotti, M. Mischi, and R. Sluijter, A robust fetal ECG detection method for abdominal recordings, Physiological Measurement, 2007, 28, 373-388.

[30] S. Bouwstra, W. Chen, L. Feijs, and S. Oetomo, Smart Jacket Design for Neonatal Monitoring with Wearable Sensors, in Body Sensor Networks (BSN 2009), Berkeley, USA, 2009, 162-167.

[31] W. Chen, I. B. I. Ayoola, S. Bambang Oetomo, and L. M. G. Feijs, Non-invasive blood oxygen saturation monitoring for neonates using reflectance pulse oximeter, in Design, Automation and Test in Europe - Conference and Exhibition 2010, (DATE 2010) Dresden, Germany, 2010.

[32] W. Chen, S. Dols, S. Bambang Oetomo, and L. M. G. Feijs, Monitoring Body Temperature of Newborn Infants at Neonatal Intensive Care Units Using Wearable Sensors, in the Fifth International Conference on Body Area Networks (BodyNets 2010), Corfu Island, Greece, 2010.

[33] W. Chen, S. T. Nguyen, R. Coops, S. Bambang Oetomo, and L. M. G. Feijs, Wireless transmission design for health monitoring at neonatal intensive care units, in the 2nd international symposium on applied sciences in biomedical and communication technologies (ISABEL 2009), Bratislava, Slovak Republic, 2009. 
[34] W. Chen, S. Bambang Oetomo, L. M. G. Feijs, P. Andriessen, F. Kimman, M. Geraets, and M. Thielen, Rhythm of Life Aid (ROLA) - An Integrated Sensor System for Supporting Medical Staff during Cardiopulmonary Resuscitation (CPR) of Newborn Infants, accepted by IEEE Transactions on Information Technology in Biomedicine, 2009.

[35] S. Bambang Oetomo, L. M. G. Feijs, W. Chen, and P. Andriessen, Efficacy of audio-promoted rate guidance for insufflation and chest compressions and feed-back signalling for the pressure of chest compressions during cardio-respiratory resuscitation (CPR) of newborn infants, in the annual meeting of the Society for Pediatric Research (SPR 2009), Baltimore, US, 2009.

[36] W. Chen, C. Sonntag, F. Boesten, S. Oetomo, and L. Feijs, A power supply design of body sensor networks for health monitoring of neonates, Intelligent Sensors, Sensor Networks and Information Processing (ISSNIP 2008), 2008, 255-260.

[37] W. Chen, C. Sonntag, F. Boesten, S. Oetomo, and L. Feijs, A design of power supply for neonatal monitoring with wearable sensors, Journal of Ambient Intelligence and Smart Environments, 2009, 1, 185-196.

[38] L. Hazelhoff, J. Han, S. Bambang-Oetomo, and P. de With, Behavioral State Detection of Newborns Based on Facial Expression Analysis, in Advanced Concepts for Intelligent Vision Systems (ACIVS), 2009, 698-709.

[39] P. J. F. Peters, L. M. G. Feijs, and S. G. Oei, Plug and play architectures for rapid development of medical simulation manikins, in the 12th World multi-conference on systemics, cybernetics and informatics (WMSCI 2008), Orlando, Florida, 2008, 2, 214-219.

[40] J. Hu and L. Feijs, A Distributed Multi-agent Architecture in Simulation Based Medical Training, Transactions on Edutainment, LNCS 5940, 2009, 3, 105-115.

[41] World Health Organization, Kangaroo mother care: a practical guide, in Department of Reproductive Health and Research. 2003.

[42] L. Sornmo and P. Laguna, Bioelectrical signal processing in cardiac and neurological applications: Elsevier Academic Press, 2005.

[43] N. Thakor and Y.-S. Zhu, Applications of adaptive filtering to ECG analysis: noise cancellation and arrhythmia detection, IEEE Transactions on Biomedical Engineering, 1991, 38, 785-794.

[44] Y. Mendelson, Pulse oximetry: theory and applications for noninvasive monitoring, Clinical chemistry, 1992, 38, 1601-1607.

[45] S. Dollberg, A. Rimon, H. Atherton, and S. Hoath, Continuous measurement of core body temperature in preterm infants, American journal of perinatology, 2000, 17, 257-264 


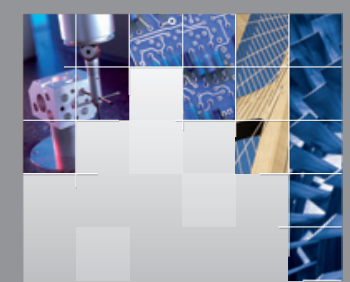

\section{Enfincering}
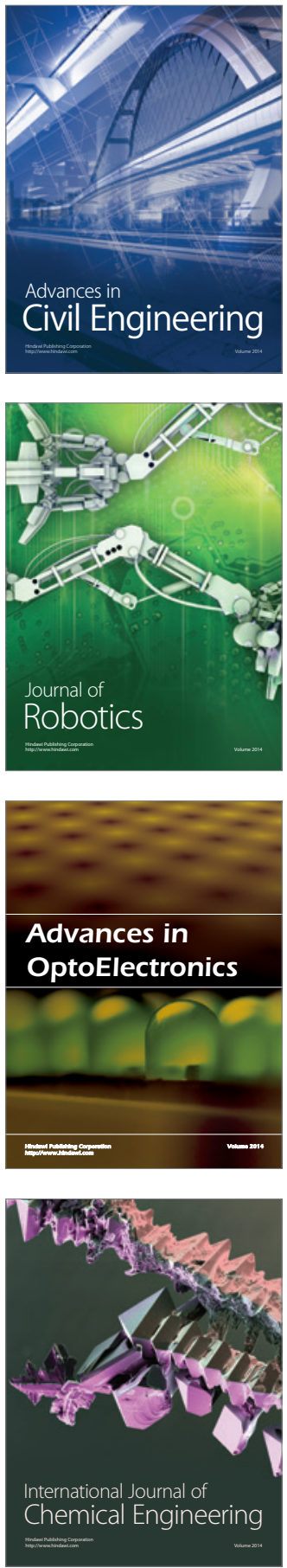

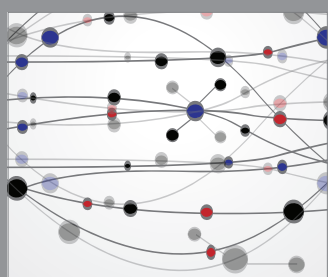

The Scientific World Journal

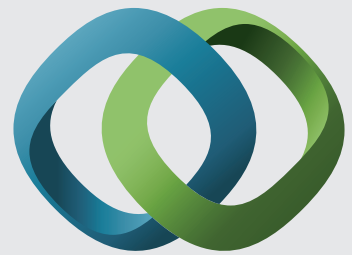

\section{Hindawi}

Submit your manuscripts at

http://www.hindawi.com
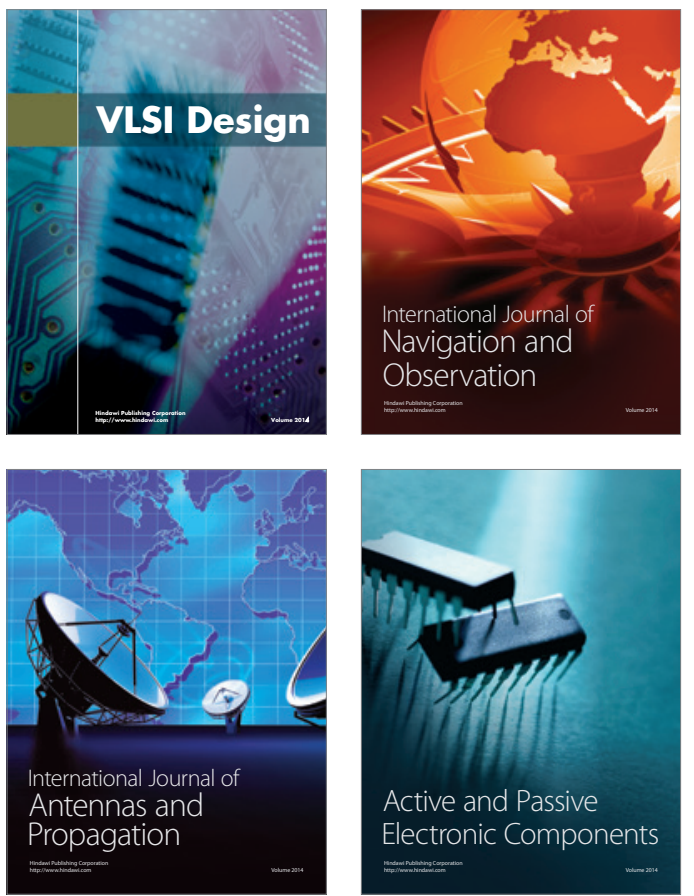
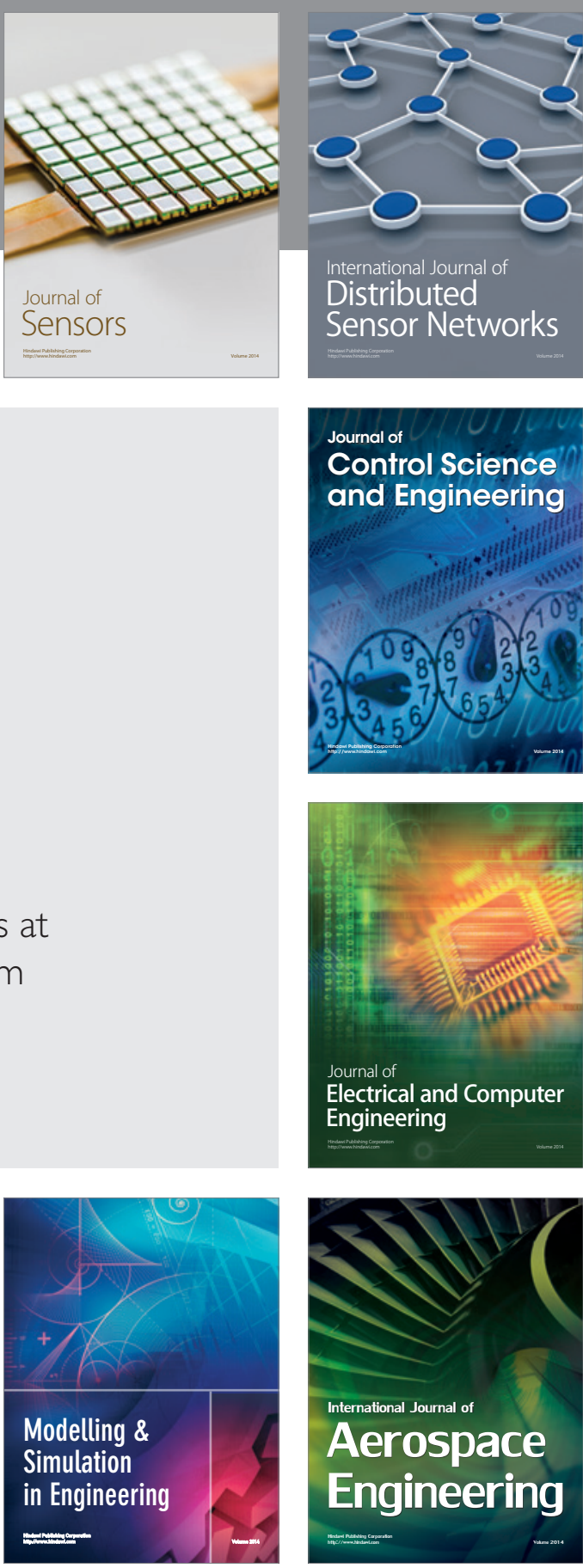

International Journal of

Distributed

Sensor Networks

Journal of

Control Science

and Engineering
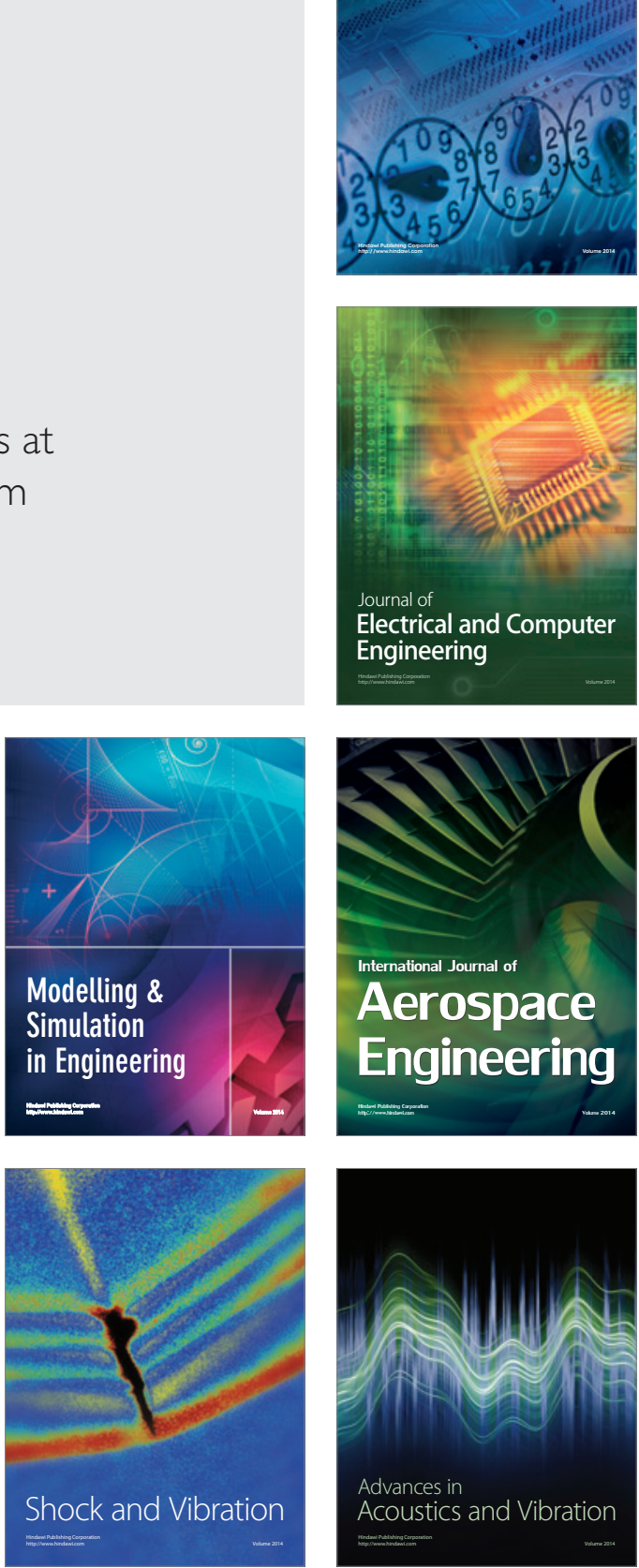